\title{
A MASSIVE BH IN THE EDGE-ON E/SO GALAXY NGC 4342
}

\author{
FRANK C. VAN DEN BOSCH \\ University of Washington, Seattle, WA 98195, USA \\ AND \\ NICOLAS CRETTON \\ Leiden Observatory, P.O. Box 9513, Leiden, The Netherlands
}

\begin{abstract}
We present evidence that the edge-on E/S0 galaxy NGC 4342 harbours a massive $\mathrm{BH}$ of $\sim 3.0 \times 10^{8} \mathrm{M}_{\odot}$. The evidence is based on a comparison of fully general three-integral models with both ground-based and HST/FOS kinematics.
\end{abstract}

The E/S0 galaxy NGC 4342 is a small low-luminosity galaxy in the Virgo cluster which harbours, in addition to its outer disc, a bright nuclear stellar disc. A combination of observations with the $4.2 \mathrm{~m}$ William Herschel Telescope (WHT) and with the Hubble Space Telescope (HST) has shown that NGC 4342 rotates rapidly and has a strong central increase in velocity dispersion (van den Bosch, Jaffe \& van der Marel 1997, see Figure 1).

We have constructed fully general three-integral models, using a modified version of Schwarzschild's orbit-superposition technique, which is based on finding the ensemble of orbits that best fits the observations. These models allow to reproduce the full line-of-sight velocity distributions and take seeing convolution and pixel binning into account (see Rix et al. 1997; van der Marel et al. 1997; Cretton et al. 1998). We constructed a range of dynamical models that differ in $\mathrm{BH}$ mass and $M / L$ only, and compared the different models with the data using $\chi^{2}$ statistics.

The models can fit all ground-based and HST data simultaneously, but only when a central BH is included. Models without BH are ruled out to a confidence level better than $\mathbf{9 9 . 7 3}$ per cent (see Figure 2). We determine a BH mass of $3.0_{-1.0}^{+1.7} \times 10^{8} \mathrm{M}_{\odot}$, where the errors are the formal 68.3 per cent confidence levels. This corresponds to 2.6 per cent of the total mass of the bulge, making NGC 4342 one of the galaxies with the highest $\mathrm{BH}$ mass to bulge mass ratio currently known. 

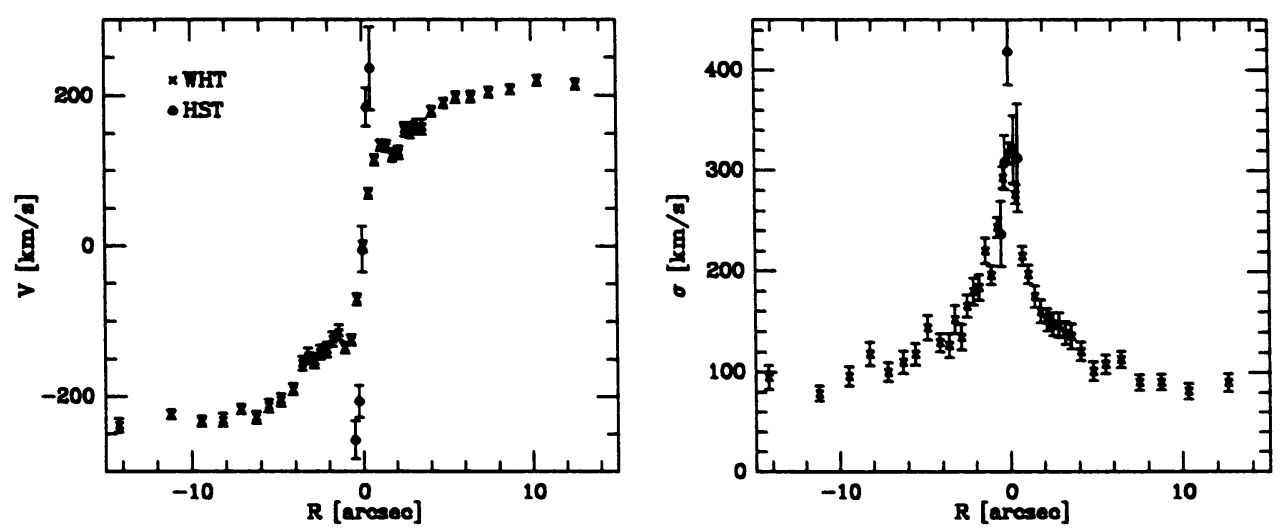

Figure 1. Rotation velocities $V$ (left) and velocity dispersions $\sigma$ (right) along the major axis of NGC 4342 obtained with the WHT (crosses) and HST/FOS (dots). The gradient of the rotation velocity and the central velocity dispersion increase considerably going to the four times higher spatial resolution of the FOS (yielding $V \approx 200 \mathrm{~km} \mathrm{~s}^{-1}$ at $0.25^{\prime \prime}$, and $\sigma_{0} \sim 420 \mathrm{~km} \mathrm{~s}^{-1}$ ).
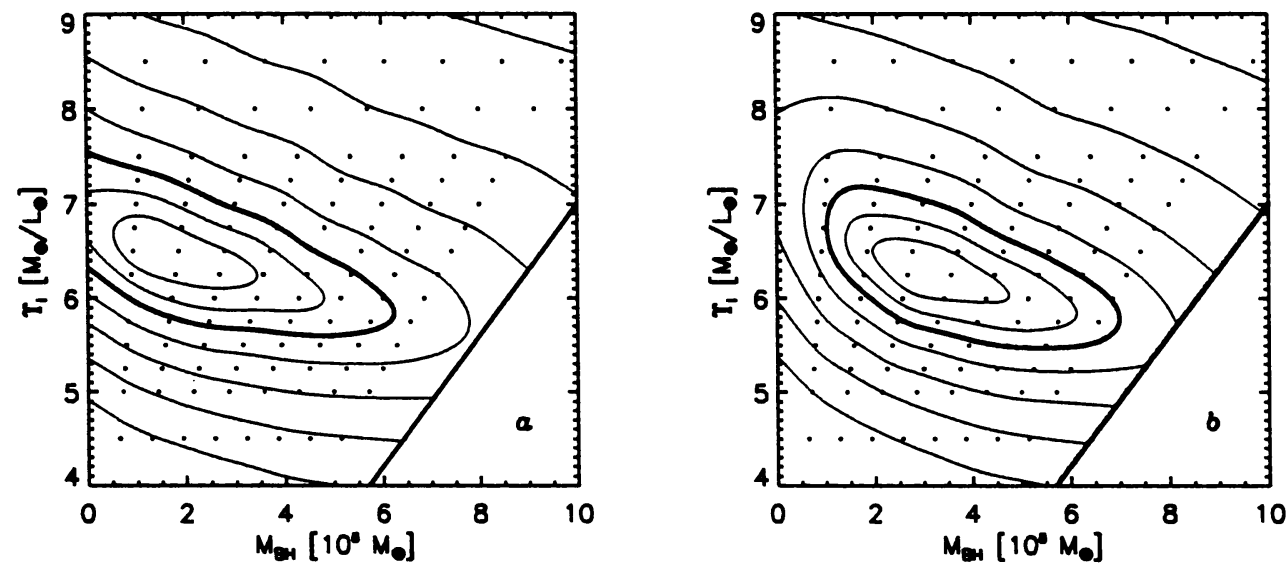

Figure 2. Contour plots of $\chi^{2}$ which measures the goodness-of-fit to the data as function of BH mass and $I$-band $M / L$-ratio. The first three contours define the formal $68.3,95.4$ and 99.73 confidence levels. Solid dots indicate actual model calculations. The $\chi^{2}$ surface of panel $a$ result from only including the WHT data. Including the HST data as well, results in the $\chi^{2}$ surface of panel $b$. Clearly, the higher spatial resolution HST data allows us to rule out models without BH to a confidence level better than 99.73 per cent.

\section{References}

Cretton N., de Zeeuw P.T., van der Marel R.P., Rix, H.-W., 1998, in prep.

Rix H.-W., et al., 1997, ApJ, 488, 702

van den Bosch F.C., Jaffe W., van der Marel R.P. 1997, MNRAS, in press

van der Marel R.P., de Zeeuw P.T., Rix H.-W., Quinlan G.D., 1997, Nature, 385, 610 